\title{
Subjective Modificatron of Aging STOCHASTIC SYSTEMS
}

\author{
By T..Y. Kam ${ }^{1}$ and Colin B. Brown ${ }^{2}$
}

\begin{abstract}
The overall uncertainty of an aging stochastic system is analyzed through the consideration of both objective uncertainty and subjective imprecision. The updated probability distribution of a normal variate is determined through the constructions of global and local entropy equations. A subjective pairwise comparison method is applied in transforming subjective information concerning the overall uncertainty of the stochastic system to fuzzy support. A comparison of two fuzzy entropy functions is made. Since the fuzzy entropy function is not unique, calibration on the actual probability distribution is needed in order to justify the use of the proposed expressions. However, in many practical situations it is possible to make sensible pairwise comparisons without being able to make sensible parameter estimates. In such circumstances the proposed scheme will be of value.
\end{abstract}

\section{INTRODUCTION}

In the estimate of the safety of existing buildings, it is essential to have current probability distributions for the random variables such as loadings and resistance. This suggests that a building be modeled as a stochastic system. At the time of the construction of the building, which is defined as the initial state, the probability distributions of the variables usually can be determined from statistics. As time elapses, the resistance of the building and the loads acting on it change stochastically. In order to have a reliable estimate of the safety of the building in the current state, updated probability distributions of the variables need to be determined in the light of new information concerning the changes in the system. This new information usually cannot be measured in an objective way, but can be estimated in an imprecise and subjective manner. It has been shown that subjective information can be integrated into objective probability distributions to give fuzzy probability distributions of a continuous variable through the use of fuzzy set theory and the construction of entropy equations (2).

The approach in this paper is to determine the support for a current parameter of a distribution being equal to the initial value and then using this information to generate the current parameter value. The support is obtained from Saaty's work (3) on the evaluation of relative importance of a set of objects. Entropy arguments are used in order to describe current distributions. It is assumed that the initial and final sta-

\footnotetext{
${ }^{1}$ Assoc. Prof., Dept. of Civ. Engrg., National Chiao Tung Univ., Taiwan; formerly, Post-Doctoral Fellow, Univ. of Washington, Seattle, Wash.

${ }^{2}$ Prof. of Civ. Engrg., Univ. of Washington, Seattle, Wash.

Note.-Discussion open until October 1, 1984. To extend the closing date one month, a written request must be filed with the ASCE Manager of Technical and Professional Publications. The manuscript for this paper was submitted for review and possible publication on September 15, 1982. This paper is part of the Journal of Engineering Mechanics, Vol. 110, No. 5, May, 1984. CASCE, ISSN 0733-9399/84/0005-0743/\$01.00. Paper No. 18775.
} 
tistics are known and that the initial, current and final distributions are normal.

Construction of Fuzzy Supports.-The approach of Saaty (3) is used to determine subjectively the relative weights to be assigned to members of a set with respect to an objective. This involves pairwise comparisons between $n$ such members on a scale of 1-9 and the construction of a square, $n \times n$, matrix, $A$, with the reciprocal property of $a_{i j}=a_{j i}^{-1}$. The weights, $W_{i}(i=1-n)$ appear in the resulting characteristic equation $(A$ $-\lambda I) W=0$, in which the values of the weights in $W$ correspond to the eigenvector corresponding to the largest eigenvalue.

In the stochastic system under consideration, weights for the two experience states (initial and final) and the current state with respect to a measure of uncertainty (say standard deviation) can be determined by this method of pairwise comparison. As a final step, the fuzzy support of the current state is obtained by a transformation (in this case a linear one) between the weights and the supports when the fuzzy supports of the initial and final states are set to 1 and 0 , respectively.

The application of this method to determine fuzzy supports of a stochastic system is shown in Example 1.

Example 1.-The determination of fuzzy supports for the states of a beam with respect to its strength.

A beam can be modeled as a stochastic system. In the initial state, the beam is considered to be in good condition and the variation of its strength can be determined from statistics. When the beam can no longer carry any loads due to damage, corrosion and cracking, it is assumed to be in the final state and the variation of its strength becomes zero. To estimate the relative weights of the current and the two extreme states of the beam with respect to the degree of variation of the beam's strength, pairwise comparisons among the three states are performed. The question posed is: Given two states, how much larger is the variation of the first one compared to that of the second? The results are shown in Table 1. For example, the first row of Table 1 gives the pairwise comparison of the variation of the initial state with the other two. It is of equal variation to itself and between weak and equal variation when compared with the current state, therefore, the value 2 in the second position, and of very large variation when compared with the final state, therefore, the value 9 in the third position (Saaty provides some help in making these assessments). The reciprocal values of the aforementioned entries are put in the transpose position and the process continued for the second row where the first term is now committed.

The determination of the weights involves the solution of the eigenvalue problem

TABLE 1.-Pairwise Comparison Matrix of a Beam

\begin{tabular}{c|c|c|c}
\hline $\begin{array}{c}\text { States } \\
(1)\end{array}$ & Initial & $\begin{array}{c}\text { Current } \\
(3)\end{array}$ & $\begin{array}{c}\text { Final } \\
(4)\end{array}$ \\
\hline Initial & 1 & 2 & 9 \\
Current & $1 / 2$ & 1 & 7 \\
Final & $1 / 9$ & $1 / 7$ & 1 \\
\hline
\end{tabular}




$$
\left[\begin{array}{ccc}
1-\lambda & 2 & 9 \\
1 / 2 & 1-\lambda & 7 \\
1 / 9 & 1 / 7 & 1-\lambda
\end{array}\right]\left\{\begin{array}{l}
W_{1} \\
W_{2} \\
W_{3}
\end{array}\right\}=0
$$

in which $\lambda=$ the eigenvalues and $W_{i}=$ weights. The largest eigenvalue is $\lambda_{\max }=3.02$, and the corresponding eigenvector is

$$
\left\{\begin{array}{l}
W_{1} \\
W_{2} \\
W_{3}
\end{array}\right\}=\left\{\begin{array}{l}
1.0 \\
0.58 \\
0.09
\end{array}\right\}
$$

By linear transformation the supports of the three states are $f_{i}=$ Initial state $=1 ; f_{c}=$ Current state $=0.54$; and $f_{f}=$ Final state $=0$. These are the supports for the initial, current and final states having the strength variation as the initial state.

Entropies of States.-Consider a stochastic system with an underlying variable $X$. It is assumed that $X$ has a normal distribution in the initial state and that $X$ remains normal throughout the lifetime of the system until it becomes deterministic in the final state. The probability distribution for the initial state is assumed to be known as

$p(x)=\frac{1}{\sqrt{2 \pi} \sigma} \exp \left[-\frac{1}{2}\left(\frac{x-m}{\sigma}\right)^{2}\right] ;-\infty \leq x \leq \infty$

in which $m=$ expected value; and $\sigma=$ standard deviation. In the final state, $x$ is set to $=0$, and the corresponding distribution is represented by a spike. The distributions of the two extreme states are shown in Fig. 1.

The measure of uncertainty in the initial state can be evaluated by using Shannon's information entropy (4) expressed as

$E_{i}=-\int p(x) \ln p(x) d x$

The substitution of Eq. 3 into Eq. 4 gives

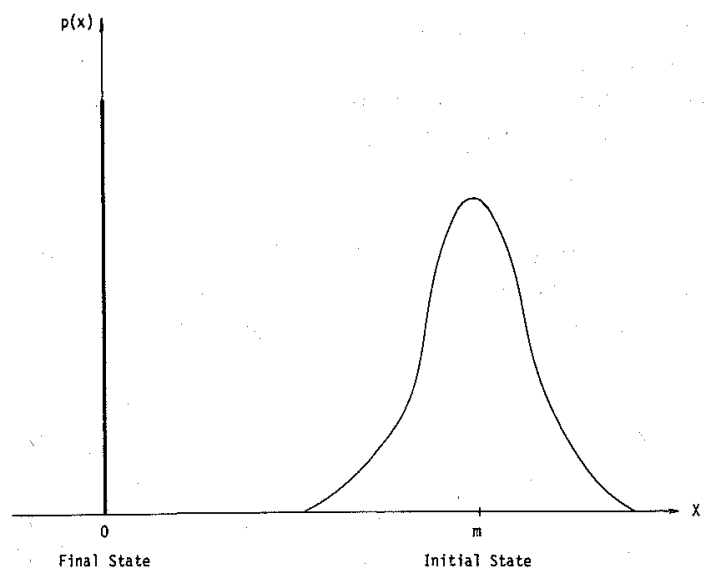

FIG. 1.-Distrlbutlons of Normal Varlate $X$ In Extreme States 
The use of continuous distributions leaves the entropy as a relative measure of uncertainty. Shannon has suggested the use of a uniform distribution over a unit interval as the separation between positive and negative entropy. This zero entropy is attained in a normal distribution when $\sigma=1 / \sqrt{2 \pi e}$. At that stage the variable approaches deterministic and this will be used as an approximation to our final state. Then

$E_{f}=0$

Probability Distribution of the Current State.-As the stochastic system ages from the initial state to the final state, it is subjectively assumed that the uncertainty in the system, compared to that of the initial state, monotonically decreases. This means that the subjective support for $\bar{\sigma}$ and $\bar{m}$ being the parameters at the current state is lower than that for $\sigma$ and $m$ at the initial state. The probability distribution of the current state can be expressed as

$$
\bar{p}(x)=\frac{1}{\sqrt{2 \pi} \bar{\sigma}} \exp \left[-\frac{1}{2}\left(\frac{x-\bar{m}}{\bar{\sigma}}\right)^{2}\right] ; \quad-\infty \leq x \leq \infty \ldots \ldots \ldots \ldots \ldots
$$

in which $\bar{m}=$ altered expected value; $\bar{\sigma}=$ altered standard deviation; and in which $\bar{\sigma}<\sigma$ of the initial state. The current probability distribution can be determined by the construction of objective and subjective entropies as proposed by Brown (1).

Subjective information concerning changes in the uncertainty of the system, compared to the initial state, is then sought and expressed in the form of a fuzzy set. The subjective entropy, which is a measure of the imprecision of the subjective information, can then be determined from entropy functions which satisfy at least three desiderata. The summation of both the subjective entropy and the entropy of the initial state leads to the estimated probability distribution for the current state. Two kinds of uncertainty analyses will be presented for the determination of the current probability distribution.

Analysis of Global Uncertainty.-In the current state, a measure of the overall uncertainty of the variable can be made by the construction of an entropy equation which includes measures of both objective uncertainty and subjective imprecision

$E=H+K G$

in which $E=$ the total entropy; $H=$ the objective entropy; $G=$ the subjective entropy; and $K=$ a multiplier. The objective entropy in Eq. 8 is defined as the entropy in the initial state which can be determined from statistics. Therefore, from Eq. 5

$H=\ln (\sqrt{2 \pi e} \sigma)$

The subjective part of Eq. 8 represents the measure of imprecision concerning the change of uncertainty in the system as it ages from the initial state to the current state. Let $A$ be a fuzzy set defined on the standard deviation of the variable in the current state being the same as in the initial state: 
in which $f=$ the membership support for the current standard deviation; and $\bar{\sigma}=$ to $\sigma$. When $f=1$, there is complete support for the outcome of the variable in the current state being as uncertain as in the initial state, and when $f=0$, the statistics obtained in the initial state are entirely irrelevant to the uncertainty of the current state. The determination of $f$ can be made by using Saaty's pairwise comparison method as described previously. For a support $f$, there exists an entropy function $G$ which satisfies the following three desiderata: (1) $G=0$, if and only, $f=1$; (2) $G$ must be a maximum, if and only $f=0$; and (3) $G \geq \tilde{G}$ when $\tilde{f} \geq f$. Two possible expressions for $G$ will be introduced to illustrate their effects on the altered parameters of a normal variate. They are defined as

$$
G_{1}=-\frac{1}{2-f} \ln \frac{1}{2-f}
$$

and $G_{2}=(2-f) \ln (2-f)$

If the entropy function of Eq. $11 a$ is used in the analysis, then Eq. 8 can be expressed as

$E=H+K G_{1}$

The multiplier $K$ in Eq. 12 can then be determined from the criterion that, when $f=0, E=E_{f}=0$. Then

$$
K=\frac{H}{\frac{1}{2} \ln \frac{1}{2}}
$$

The construction of $\bar{\sigma}$ is such that

$E=-\int \bar{p}(x) \ln \bar{p}(x) d x$

or from Eq. 5

$E=\ln (\sqrt{2 \pi e} \bar{\sigma})$

The substitution of Eqs. 9, 11a, 13 and 15 into Eq. 12 leads to

$\bar{\sigma}=\sigma \exp \left[-\frac{\ln (\sqrt{2 \pi e} \sigma)}{\frac{1}{2} \ln \frac{1}{2}}\left(\frac{1}{2-f} \ln \frac{1}{2-f}\right)\right]$

If the entropy function of Eq. $11 b$ is used, then Eq. 12 becomes

$E=H+K G_{2}$

and the multiplier can be determined from the same criterion as

$K=-\frac{H}{2 \ln 2}$.

The substitution of Eqs. 9, 11b, 18 and 15 into Eq. 17 gives 
$\bar{\sigma}=\sigma \exp \left[-\frac{\ln (\sqrt{2 \pi e} \sigma)}{2 \ln 2}((2-f) \ln (2-f))\right]$

A comparison of Eq. 16 and Eq. 19 is made by setting the initial standard deviation $\sigma=500$. The ratios $\bar{\sigma} / \sigma$ obtained from Eqs. 16 and 19 are plotted against membership support, $f$, in Fig. 2 as curves A and B, respectively.

From the aforementioned global uncertainty analysis, the altered standard deviation of the distribution can be determined from either Eq. 16 or Eq. 19. The altered expected value of the distribution can frst be approximated by linear interpolation. For example, if the weights obtained from Saaty's pairwise comparison method for the initial, current and final states are $W_{1}, W_{2}$ and $W_{3}$, respectively, then the first approximation of the altered expected value is

$m^{\prime}=\frac{W_{2}-W_{3}}{W_{1}-W_{3}} \cdot m$

If linear transformation has been used for the construction of support function, then Eq. 20 becomes

$m^{\prime}=f \cdot m$

Improvement on the approximation of the altered expected values can be made by performing the following local uncertainty analysis.

Analysis of Local Uncertainty.-If the parameters obtained in the foregoing global analysis are used for the current probability distribution of the variable, the probability of any event about the outcome of the variable can be evaluated. Let $\bar{x}$ be a specific value of the variable. Then the probability of $x$ being greater than $\bar{x}$ is
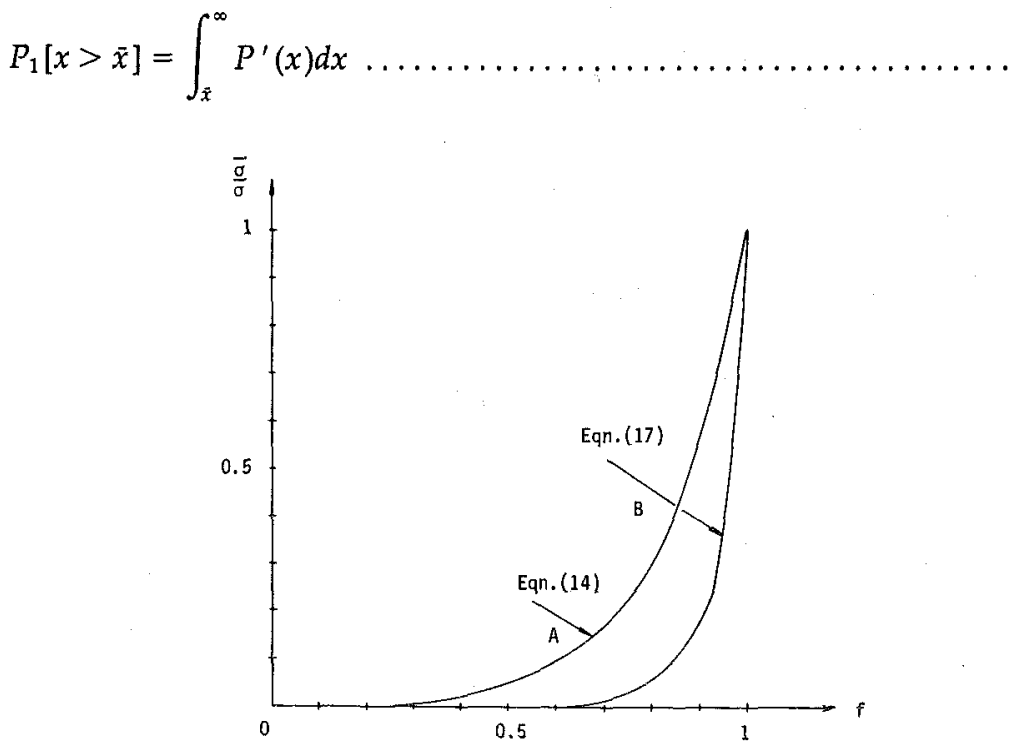

FIG, 2x-The Ratio $\bar{\sigma} / \sigma$ for Two Entropy Functions 
in which $P^{\prime}(x)=\frac{1}{\sqrt{2 \pi} \bar{\sigma}} \exp \left[-\frac{1}{2}\left(\frac{x-m^{\prime}}{\bar{\sigma}}\right)^{2}\right]$

and the probability of $x$ being smaller than or equal to $\bar{x}$ is

$P_{2}[x \leq \bar{x}]=1-P_{1}$

The objective entropy of the outcome of the variable for the posed problem is

$H_{L}=-P_{1} \ln P_{1}-P_{2} \ln P_{2}$

Subjective confidence in the proposed probabilities of the two states, i.e., $x>\bar{x}$ and $x \leq \bar{x}$, is sought, and this information is then expressed in terms of fuzzy supports $f_{1}$ and $f_{2}$. If the entropy function of Eq. 11a is used then the measure of imprecision of the subjective information is

$G_{L}=P_{1}\left(-\frac{1}{2-f_{1}} \ln \frac{1}{2-f_{1}}\right)+P_{2}\left(-\frac{1}{2-f_{2}} \ln \frac{1}{2-f_{2}}\right)$

The combined entropy for the problem is

$E_{L}=H_{L}+K_{L} G_{L}$

in which $K_{L}$ is a multiplier. The altered probabilities $\tilde{P}_{1}$ and $\tilde{P}_{2}$ are constructed such that

$E_{L}=-\tilde{P}_{1} \ln \tilde{P}_{1}-\tilde{P}_{2} \ln \tilde{P}_{2}$

in which $\tilde{P}_{2}=1-\tilde{P}_{1}$ and the criterion for the multiplier, $K_{L}$, could be that if $f_{1}=f_{2}=0$, then $\tilde{P}_{1}=\vec{P}_{2}=1 / 2$. This leads to

$$
K_{L}=\frac{\ln \frac{1}{2}+H_{L}}{\frac{1}{2} \ln \frac{1}{2}}
$$

The evaluation of $\tilde{P}_{1}$ results from Eqs. 27 and 28 . When the altered probability, $\tilde{P}$, is available, the altered expected value, $\bar{m}$, can be estimated from the relation

$\tilde{P}_{1}=1-\int_{-\infty}^{\hat{x}} \frac{1}{\sqrt{2 \pi} \bar{\sigma}} e^{-\left[1 / 2(x-\bar{m} / \bar{\sigma})^{2}\right]} d x$

If the entropy function of Eq. $11 a$ is used, Eq. 26 for measuring imprecision becomes

$G_{L}=P_{1}\left(2-f_{1}\right) \ln \left(2-f_{1}\right)+P_{2}\left(2-f_{2}\right) \ln \left(2-f_{2}\right)$

and the multiplier $K_{L}$ in Eq. 29 is

$K_{L}=\frac{-\ln \frac{1}{2}-H_{L}}{2 \ln 2}$

The altered probabilities are obtained from Eqs. 27 and 28 in the light 
of Eqs. 24, 25, 31 and 32, and the altered expected value is determined from Eq. 30.

The application of the global and local uncertainty analyses in determining the altered parameters of a normal variate will be illustrated in Example 2.

Example 2.-Consider the beam given in Example 1. Assume the strength of the beam to be described by a normal variate, $X$. In the initial state, the two parameters of the distribution are defined as $\sigma=500$ kips, and $m=5,000$ kips, and in the final state, the strength of the beam becomes zero. The probability distribution of the initial state is shown in Fig. 3 as curve A. If the support for the global uncertainty of the strength of the beam in the current state from Saaty's method is

$f=0.54$

then the altered standard deviation can be determined from either Eq. 16 or Eq. 19. If the entropy function of Eq. $11 a$ is used in the analysis then from Eq. 16 it gives

$\bar{\sigma}=1.7$ kips

The first approximation for the altered expected value determined from Eq. 21 is

$m^{\prime}=f \cdot m=2,700 \mathrm{kips}$

In the local analysis, if $\bar{x}$ is chosen as $\bar{x}=m^{\prime}-2 \bar{\sigma}$ or $\tilde{x}=2,696.6$ kips and the fuzzy supports for the two states, $x>2,696.6$ kips and $x \leq$ $2,696.6$ kips are $f_{1}=0.5$ and $f_{2}=0.5$, respectively, then the altered ex-

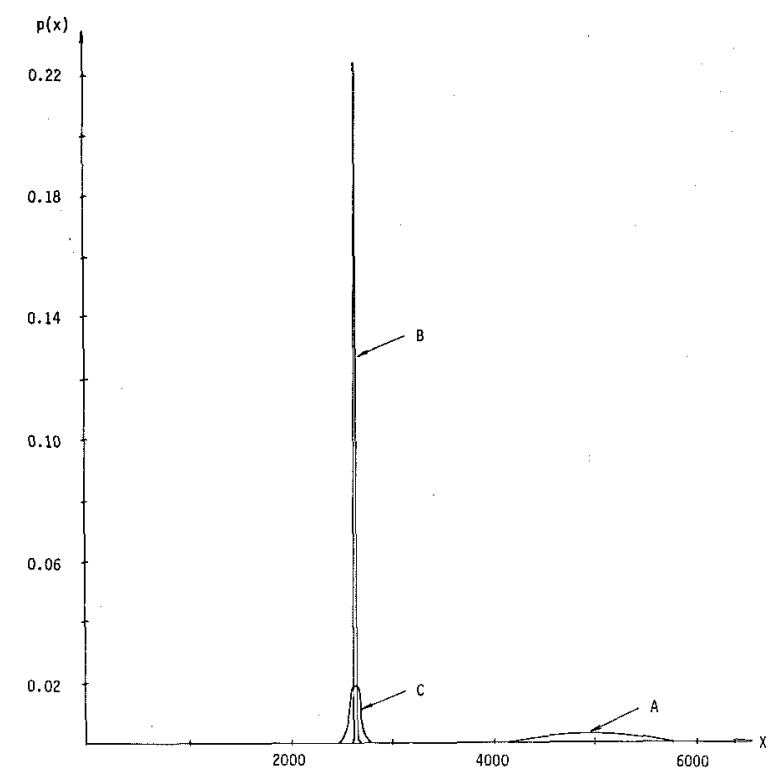

FIG. 3.- Probability DIstribution of Beam Strength in Initial and Current States 
pected value obtained from $\mathrm{Eq} .30$ is

$\tilde{m}=2,699$ kips

The probability distribution of the strength of the beam with updated parameters is shown in Fig. 3 as curve B.

If the entropy function of Eq. $11 b$ is used in the aforementioned analysis then the altered standard deviation obtained from Eq. 19 is

$\bar{\sigma}=24$ kips

and from the local analysis it gives

$\bar{m}=2,672$ kips

The corresponding probability distribution is shown in Fig. 3 as curve C.

Review.-The scheme proposed allows an investigator to use subjective pairwise comparisons between known and current states as input to produce specific current parameters as outputs of the analysis. These results may then be examined for sensibility. This procedure will not be of interest when direct indicators of parameters are available. However, in many practical situations it is possible to make sensible pairwise comparisons without being able to make sensible parameter estimates. In such circumstances the proposed scheme will be of value.

\section{CONCLUSION}

The overall uncertainty of an aging stochastic system has been analyzed through the consideration of both objective uncertainty and subjective imprecision. The updated probability distribution of a normal variate has been determined through the constructions of global and local entropy equations. Saaty's pairwise comparison method has been applied in transforming subjective information concerning the overall uncertainty of the stochastic system to fuzzy support. A comparison of two fuzzy entropy functions has been made. Since the fuzzy entropy function is not unique, calibration on the actual probability distribution is needed in order to justify the use of the proposed expressions.

\section{ACKNOWLEDGMENT}

This work was supported by the National Science Foundation (Grant No. CME-802 6 583).

\section{Appendix.-References}

1. Brown, C. B., "Entropy Constructed Probabilities," Journal of the Engineering Mechanics Division, ASCE, Vol. 106, No. EM4, Paper 15635, Aug., 1980, pp. 633-640.

2. Kam, T. Y., and Brown, C. B., "Updating with Fuzzy Entropies."

3. Saaty, T. L., "A Scaling Method for Priorities in Hierarchical Structures," Journal of Mathematical Psychology, Vol. 15, 1977, pp. 234-281.

4. Shannon, C. E., "The Mathematical Theory of Communication, Univ. of Illinois Press, Ill., 1949. 